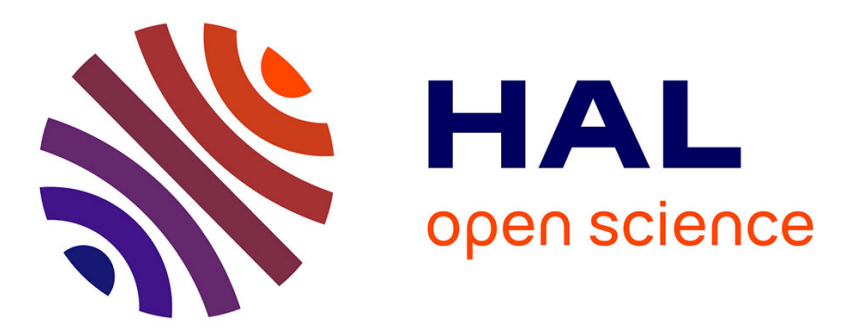

\title{
Phase unwrapping for dual-beam electronic speckle pattern interferometry: method
}

S Yoshida, Dr. Suprapedi, R. Widiastuti, Dr. Marincan, Dr. Septriyanti, Dr. Julinda, A. Faisal, A. Kusnowo

\section{- To cite this version:}

S Yoshida, Dr. Suprapedi, R. Widiastuti, Dr. Marincan, Dr. Septriyanti, et al.. Phase unwrapping for dual-beam electronic speckle pattern interferometry: method. Applied optics, 1997, 36 (1), pp.266270. hal-01092321

\section{HAL Id: hal-01092321 \\ https://hal.science/hal-01092321}

Submitted on 8 Dec 2014

HAL is a multi-disciplinary open access archive for the deposit and dissemination of scientific research documents, whether they are published or not. The documents may come from teaching and research institutions in France or abroad, or from public or private research centers.
L'archive ouverte pluridisciplinaire HAL, est destinée au dépôt et à la diffusion de documents scientifiques de niveau recherche, publiés ou non, émanant des établissements d'enseignement et de recherche français ou étrangers, des laboratoires publics ou privés. 


\title{
Phase unwrapping for dual-beam electronic speckle pattern interferometry: method
}

\author{
S. Yoshida, Suprapedi, R. Widiastuti, Marincan, Septriyanti, Julinda, \\ A. Faisal, and A. Kusnowo
}

\begin{abstract}
A new algorithm to reveal the order and sign of fringes formed by dual-beam electronic speckle-pattern interferometry is described. When combined with a previously reported phase-extraction method, it makes possible the evaluation of displacement absolutely and continuously over the entire surface of the object from a total of five frames. This technique is particularly useful when displacement is spatially discontinuous and temporally fast and the amount of data is large. A model experiment showing the validity of this method is presented.
\end{abstract}

We have applied dual-beam, inplane sensitive electronic speckle pattern interferometry ${ }^{1}$ (ESPI) to the study of plastic deformation. The key subject of this study is an investigation of the temporal change in the spatial distribution of distortion tensor components (strain and rotation) in connection with a material fracture. ${ }^{2}$ As described in detail in Ref. 3 , the spatial distribution of distortion tensor components varies both spatially and temporally in a complicated way, depending on the material's substructure, which also changes temporally as the deformation progresses. It is essential to obtain fringe patterns sequentially with an interval sufficiently small to trace abrupt change in the material's mechanical state in the plastic region, which obliges us to handle a large amount of image data. In the currently conducted tensile analysis, we typically acquire more than 200 sets of image data every $10 \mathrm{~s}$. Because the mechanical state of the object changes every minute, it is particularly important to take image data corresponding to the same state, i.e., the state before or after a displacement of interest, at a sharply defined moment. In the case of a tensile load the material changes its mechanical state at a rate higher than the

The authors are with the Research and Development Center for Applied Physics, Puspiptek, Serpong, Tangerang 15310. Indonesia. head speed of the tensile machine by an order of magnitude or even higher. ${ }^{4}$ If the head speed is 0.35 $\mathrm{mm} / \mathrm{min}$, for example, this rate becomes $3.5 \mathrm{~mm} /$ $\min =58 \mu \mathrm{m} / \mathrm{s}$, and this means that if the uncertainty in the data acquisition time is $0.01 \mathrm{~s}$ (the order of typical scanning time of a video frame), the resulting error in displacement becomes $0.58 \mu \mathrm{m}$, which is comparable with the wavelength of the $\mathrm{He}-\mathrm{Ne}$ laser, the laser used in our optical setup.

In this condition, it is unrealistic to employ conventional phase-step methods 5,6 to extract displacement from image data. First, the phase-step method requires at least two frames both before and after displacement. ${ }^{5}$ It then uses these images to calculate the absolute value of the displacement. Therefore, unless all the frames corresponding to the same state, i.e., before or after displacement, are taken within a time negligibly smaller than the above-mentioned time scale of the temporal change of the mechanical state, the resultant displacement data do not correspond to two sharply defined states. Hence they become meaningless. Second, because the phase-step methods evaluate the phase in the range of $-\pi$ to $\pi$, phase unwrapping is needed as a post-dataacquisition process. We usually do this by presuming the unknown multiple of $2 \pi$ for each pixel in such a way that the resultant phase difference between adjacent pixels may not be greater than $\pi .^{6}$ However, this is not suitable in our case because the spatial phase change can be discontinuous by the nature of plastic deformation.

The carrier fringe method, which is another conventional technique for extracting phase data from 


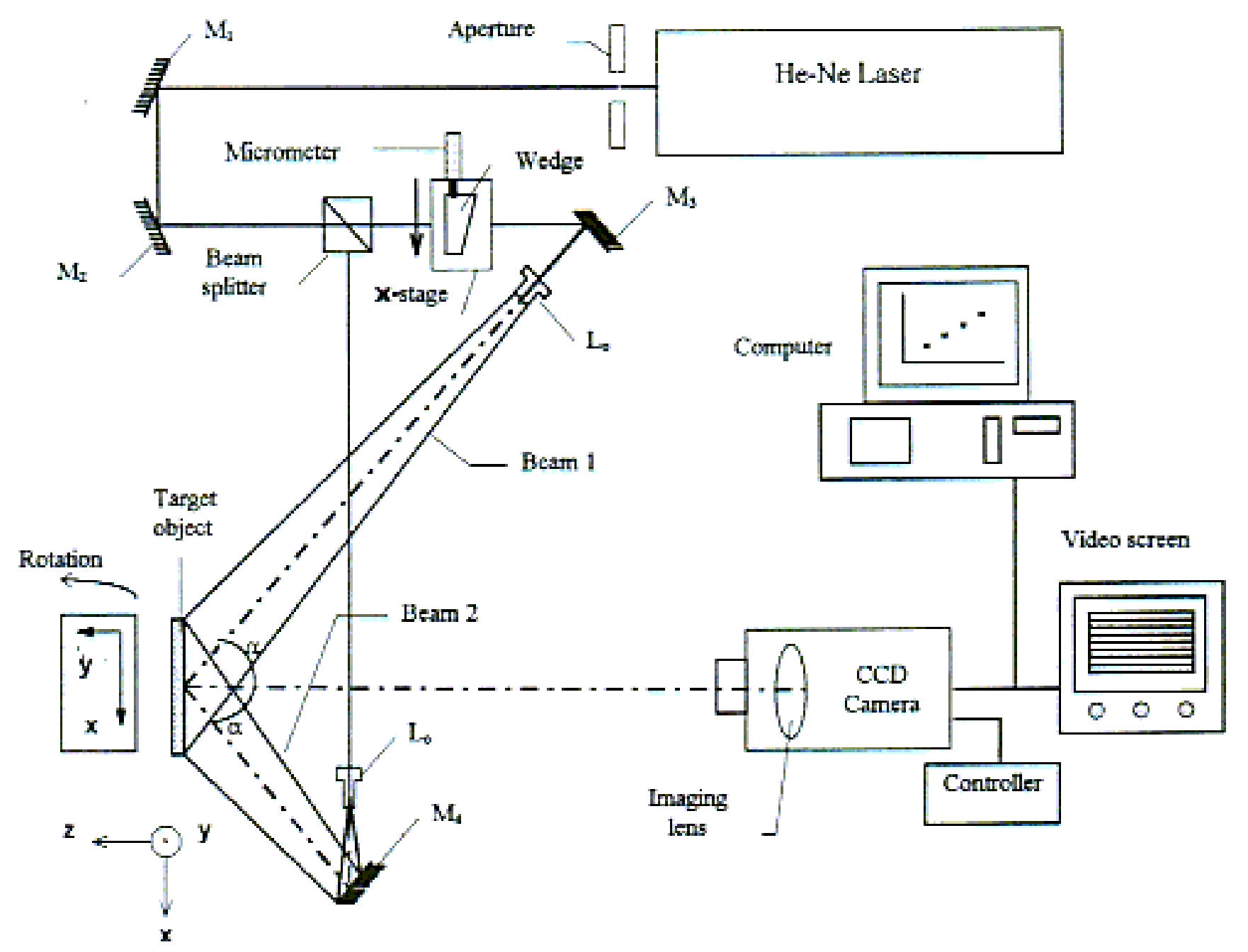

Fig. 1. Optical arrangement of the current scheme.

ESPI fringe patterns capable of evaluating displacement corresponding to two sharply defined moments, is also difficult to employ in the current situation because the amount of image data is too large for us to conduct a post-data-acquisition process to filter out the carrier-fringe frequency component from the signal component. ${ }^{7}$

For reasons mentioned above, we invented a new method of phase extraction that allows us to extract displacement corresponding to two definite times. ${ }^{8}$ In short, this method (referred to as the new phaseextraction method) functions in the following way. At each time step, four frames of image are taken, two of which correspond to the states before and after the current displacement, and the other two correspond to the average intensities of the respective interferometric branches. Because the latter two are irrelevant to the phase change and therefore considered to be constant during the displacement, the extracted displacement data correspond to two sharply defined states. However, because the new phase-extraction method outputs the phase data in the form of an arctangent of an intensity ratio,$^{8}$ ambiguity concerning the order and sign of fringes remains. The aim of this paper is to introduce an algorithm to clarify this ambiguity.

Our idea is to extract the absolute value of the phase by the new phase-extraction method and reveal the order and sign of fringes by monitoring the directions in which the fringes move when a phase shift is introduced into one of the interferometric branches. Note that, although the current method resembles the phase-step method in the sense that one of the interferometric branches is phase shifted, the current method is fundamentally different in the fact that it does not use the phase-shifted image data for evaluation of the absolute value of the phase but for determining the order and sign of fringes only. As described below, the direction of the fringe movement is solely dependent on the spatial derivative (slope) of the phase data along an axis of interest. It is reasonable to consider that the sign of the slope is kept the same during the phase-shift operation. In other words, it is unlikely that the relative order of neighboring fringes is reversed in such a short time. (At least it can be said that the sign of the slope varies more slowly than the absolute value of phase.) Because the fringe shift is used only to determine the sign of the slope, the amount of the phase shift given to one of the branches does not have to be known quantitatively as long as it is known to be less than a quarter of the period so that the direction of the fringe shift may be known. Thus a simple means such as an optical wedge can be used for the phase shifter, and no computation is needed. The present algorithm can be used to reveal the order and sign of fringes formed by dualbeam ESPI in general.

Consider Fig. 1, which illustrates an inplane sensitive ESPI setup ${ }^{1}$ and the phases of beam 1 and beam 2 at a reference point on the object surface. Suppose the object deforms and the reference point is displaced by $u$ in the positive $x$ direction (leftward). If the initial phases of beam 1 and beam 2 at the reference point are $\theta_{10}$ and $\theta_{20}$, respectively, and the displacement causes a phase change of $\phi / 2$, the resultant phases of these beams after the deformation 
are

$$
\begin{aligned}
& \theta_{1}=\theta_{10}+\phi / 2, \\
& \theta_{2}=\theta_{20}-\phi / 2,
\end{aligned}
$$

and the phase difference becomes

$$
\theta=\theta_{1}-\theta_{2}=\theta_{0}+\phi,
$$

where $\theta_{0}$ is the initial phase difference, $\theta_{10}-0_{20}$, and $\phi$ is defined to be positive when the displacement $u$ is in the positive direction. The phase change $\phi / 2$ is related to the displacement $u$ through a constant of proportion $2 \pi \sin (\alpha) / \lambda$ as

$$
\phi / 2=2 \pi u \sin (\alpha) / \lambda,
$$

where $\alpha$ is the angle of incidence and $\lambda$ is the wavelength of the laser.

In this situation, let us increase the phase of beam 1 at the reference point by $\delta_{w}$, where $0<\delta_{w}<\pi / 4$. This can be done, for example, by moving a wedge placed in the optical path of beam 1 in such a way that the optical path in the wedge becomes longer by $\delta_{w}$ inphase (Fig. 1). This changes the phase of beam 1 at the reference point to

$$
\theta_{1}{ }^{\prime}=\theta_{10}+\phi / 2+\delta_{i k},
$$

whereas the phase of beam 2 is unchanged. Consequently the phase difference becomes

$$
\theta^{\prime}=\theta_{1}{ }^{\prime}-\theta_{2}=\theta_{0}+\phi+\delta_{u} \text {. }
$$

Now consider the subtraction of the images taken before and after the deformation. Let us call the images taken before the deformation $I_{\text {bef }}$, after the deformation but before the wedge movement $I_{\text {aft, }}$ and after the deformation and wedge movement $I_{\text {aft }}$. The intensities of these images can be written as

$$
\begin{aligned}
I_{\text {bef }} & =I_{0}[1+\gamma \cos (\theta)], \\
I_{\text {aft }} & =I_{0}[1+\gamma \cos (\theta+\phi)], \\
I_{\mathrm{aft}}{ }^{\prime} & =I_{0}\left[1+\gamma \cos \left(\theta^{\prime}+\phi\right)\right],
\end{aligned}
$$

where $I_{0}$ is the total average intensity and $\gamma$ is the visibility. Because the same argument for the reference point holds for any point on the object surface, the subtractions of $I_{\text {bef }}$ from $I_{\text {aft }}$ and $I_{\text {aft }}{ }^{\prime}$ can be expressed, respectively, as ${ }^{1}$

$$
\begin{aligned}
I_{s}= & I_{\text {aft }}-I_{\mathrm{bef}}=2 I_{0} \gamma \sin \left(\theta_{0}+\phi / 2\right) \sin (\phi / 2), \\
I_{s}{ }^{\prime}= & I_{\text {aft }}{ }^{\prime}-I_{\mathrm{bef}}=2 I_{0} \gamma \sin \left(\theta_{0}+\phi / 2+\delta_{u^{\prime}} / 2\right) \\
& \times \sin \left(\phi / 2+\delta_{w} / 2\right) .
\end{aligned}
$$

Note that $\theta_{0}$ and $\phi$ are functions of $(x, y)$ whereas $\delta_{w}$ is a constant.

As is well known, $I_{\delta}$ represents a fringe system in which fringes (dark peaks) appear at the locations where the displacement is such that the corresponding phase change $\phi$ satisfies the condition $\sin (\phi / 2)=$ 0 . Physically this means that if part of the object displaces in such a way that the resultant phase

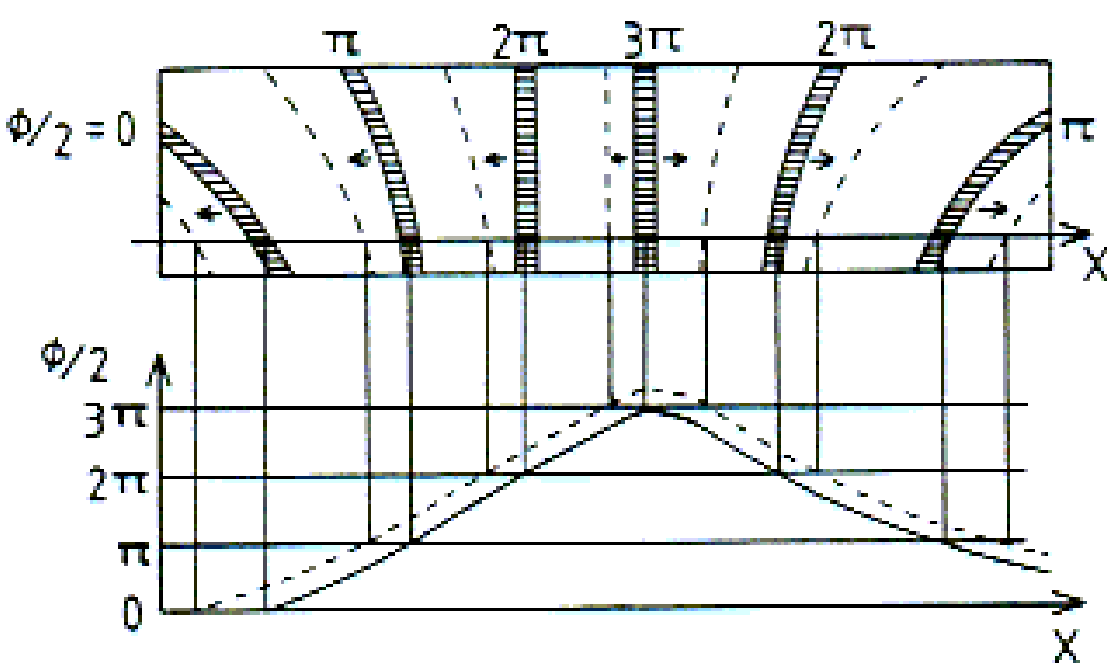

Fig. 2. $I_{x}$ and $I_{s}^{\prime}$ fringe patterns (upper) and phase variation along line $x$ (lower). Arrows indicate the directions of the fringe shift. Vertical lines relate the fringes and their phase values.

change is $\phi / 2=n \pi$, the intensity of the speckle generated by that part of the object is unchanged before and after the displacement. Now consider that such a displacement is accompanied by a phase change of $\delta_{w}$ in the first beam. In this case, those parts of the object that displace the phase changes of $\phi / 2=n \pi-$ $\delta_{r e}$, rather than $\phi / 2=n \pi$, will form fringes in the resultant subtraction image. This means that if one compares $I_{s}$ and $I_{s}{ }^{\prime}$, the fringes are found to be shifted from the loci of displacement corresponding to $\phi / 2=$ $n \pi$ to those of $\phi / 2=n \pi-\delta_{w}$ in going from $I_{s}$ to $I_{s}{ }^{\prime}$. In $I_{s}$ therefore, if the locus of $\phi / 2=n \pi-\delta_{w}$ is located to the left of the locus of $\phi / 2=n \pi$, the fringe shift is leftward, and if the locus of $\phi / 2=n \pi-\delta_{w}$ is located to the right of the locus of $\phi / 2=n \pi$, the fringe shift is rightward. The important thing is that the direction of the fringe shift is determined by the location of the $\phi / 2=n \pi-\delta_{w}$ locus relative to that of $\phi / 2=n \pi$, not by the sign of $\phi / 2$.

To make the above argument understood more easily, let us use Fig. 2. Suppose the fringe pattern representing $I_{s}$ is as shown in the upper half of Fig. 2, and the phase variation $\phi(x, y) / 2$ along a representative axis $x$ is as shown in the lower half of Fig. 2 . Because the phase value at any point in the $I_{s}{ }^{\prime}$ image is higher by $\delta_{w} / 2$ than that of $I_{s}$ at the same point [compare Eqs. (10) and (11)], the phase variation on the $I_{s}$ ' image along the same axis $x$ should be as shown by the dashed curve in the lower part of Fig. 2 . This means that the $x$ values at which fringes were seen in the $I_{s}$ image now correspond to phase values of $n \pi+\delta_{w} / 2$ in $I_{s}{ }^{\prime}$, and the $x$ values that were corresponding to $n \pi-\delta_{w} / 2$ in $I_{s}$ now correspond to fringes at $n \pi$ in $I_{s}{ }^{\prime}$. Thus the fringes in $I_{s}{ }^{\prime}$ are shifted, compared with $I_{s}$, either rightward or leftward depending on the slope of the $\phi(x, y) / 2$-versus- $x$ curve [hereafter called the $\phi(x, y) / 2$ curve] in the vicinity of the fringe positions as indicated by dashed curves in the upper part of Fig. 2.

The key point in the above argument is that the amount and direction of the fringe shift depend solely on the slope of the $\phi(x, y) / 2$ curve and the amount and direction of the wedge movement. In the case of Fig. 2 where the wedge movement increases the 
Table 1. Logic to Determine Fringe Order

\begin{tabular}{|c|c|c|c|}
\hline \multicolumn{2}{|c|}{ Sign of Slope $(\delta \phi / \delta x)$} & \multicolumn{2}{|c|}{ Phase at Fringe } \\
\hline $\begin{array}{c}\text { Fringe } \\
\text { a }\end{array}$ & $\begin{array}{c}\text { Fringe } \\
\mathrm{b}\end{array}$ & $\begin{array}{c}\text { Fringe } \\
\mathrm{a}\end{array}$ & $\begin{array}{c}\text { Fringe } \\
b\end{array}$ \\
\hline+ & + & $n \pi$ & $(n+1) \pi$ \\
\hline+ & - & $n \pi$ & $n \pi$ \\
\hline- & + & $n \pi$ & $n \pi$ \\
\hline- & - & $n \pi$ & $(n-1) \pi$ \\
\hline
\end{tabular}

phase of beam 1 (the wedge thickness increases as in Fig. 1), the fringe necessarily shifts rightward if the slope is negative and leftward if the slope is positive. The reverse is also true; i.e., if a fringe shifts leftward (rightward) the slope is necessarily positive (negative) around that fringe, provided that the direction of the wedge movement is the same. If the wedge moves in the other direction, the $\phi(x, y) / 2$ curve moves the other way and the fringe shifts to the opposite direction for the same $\phi / 2$ slope. The amount of the fringe shift indicates the distance between the two values of $x$ corresponding to $\phi(x, y) /$ $2=n \pi-\delta_{w} / 2$ and $\phi(x, y) / 2=n \pi$ and therefore is inversely proportional to the absolute value of the slope. The same argument holds along any axis.

The above-mentioned relationship between the fringe shift and the $\phi(x, y) / 2$ slope can be conveniently used to determine the order of fringes for a given fringe pattern. Let us use Fig. 2 to discuss this process. First, suppose that the $I_{s}$ and $I_{s}{ }^{\prime}$ fringe patterns are given and the orders of these fringes are unknown (therefore the $\phi / 2$ curve is unknown). By comparing $I_{s}$ and $I_{s}{ }^{\prime}$, we know that each fringe moves in which direction. The two leftmost fringes (let us call them fringes a and b), for example, are shifted leftward when the thickness of the wedge increases. From this and the argument above, we know that the slopes in the vicinities of these fringes are positive. The slopes around all the other fringes can be known in the same way.

Now that the slope of every fringe is known, we can determine the order of the fringes by the following logic. Let us consider fringes $a$ and $b$ and suppose that fringe a corresponds to $\phi / 2=n \pi$. Because the slopes of the $\phi / 2$ curve in the vicinities of both fringes are known to be positive, we know that the order of fringe $b$ is higher than $a b y$. This is because, if the order of fringe $b$ is the same or lower than that of fringe a, the $\phi / 2$ curve must cross the line $n \pi$ (corresponding to fringe a's order) at least once again in order to cross fringe $b$ with a positive slope, and if this is the case there must be another fringe appearing between fringe $a$ and $b$. In this fashion one can determine the order of a fringe relatively to a neighboring fringe based on the slopes of the $\phi / 2$ curve at these fringes. Table 1 shows the logic for the other cases.

This argument indicates that if the order of one of the fringes is absolutely known (call this fringe the reference fringe), one can determine the orders of all the other fringes by applying the logic shown in Table 1 to each fringe one by one, starting from the reference fringe. The order of the reference fringe, on the other hand, can be determined in a couple of ways: One possible way is to change the wavelength or the incident angle and determine how the fringes move. Either of these changes alters the coefficient in proportion to Eq. (4) and therefore makes all the fringes appear at different values of $u$ in $I_{s}$, except for $u=0$. Thus the fringe corresponding to $\phi / 2=0$ can be found as the only fringe insensitive to these changes. Once the orders of subtraction-mode fringes are determined, the orders of the addition-mode fringes ${ }^{8}$ [corresponding to $1 / 2(m+\pi)$, where $m$ is an integer] can be determined by the same logic, and all the phase values are evaluated without the sign and quadrant ambiguity.

To verify this phase-unwrapping method, we conducted an experiment using a model object. We used an aluminum plate for the target object and gave it a small rotation about an axis normal to its surface. This generates an equidistant parallel fringe system in which the phase change $\phi$ owing to the rotation is proportional to an axis, say the $y$ axis, perpendicular to the direction at which the interferometer is sensitive. ${ }^{8}$ This means that the $\phi$-versus- $y$ curve becomes linear and that therefore when the wedge is moved all the fringes are expected to shift by the same amount in parallel to the $y$ axis.

We mounted a wedge having a slant angle of $0.5^{\circ}$ and surface roughness of $<\lambda / 4$ at $0.6328 \mu \mathrm{m}$ on a one-dimensional movable stage (called the $x$ stage) and placed the $x$ stage in the optical path of beam 1 (Fig. 1). To place and move the wedge precisely perpendicularly to the optical path, we placed an aperture near the output mirror of the laser and adjusted the angle of the wedge to the optical path in such a way that the laser beam reflected at the input surface of the wedge may go through the aperture. Because the aperture diameter and the distance between the aperture and the wedge were approximately 5 and $3600 \mathrm{~mm}$, respectively, the error in the angle of incidence to the wedge is estimated to be less than $5 / 3600=1.39 \mathrm{mrad}=0.08^{\circ}$. The phase difference caused by this error is negligibly small compared with the phase difference introduced by the wedge movement.

We moved the wedge by rotating the micrometer attached to the $x$ stage with a unit of $10 \mu \mathrm{m}$ (hereafter called the unit wedge movement). Because the angle of the wedge is $0.5^{\circ}$, the unit wedge movement causes the optical path covered by the wedge to be changed by $10 \times \tan \left(0.5^{\circ}\right)=0.0873 \mu \mathrm{m}$. This optical path change in turn causes a phase change of $2 \pi$ $\times 0.0873 / \lambda\left(n-n_{0}\right)=2 \pi \times 0.0710$, where $n$ and $n_{0}$ are the refractive indices of the wedge material and air, which are, respectively, 1 and 1.515 in the current case.

Figure 3 shows fringe patterns of $I_{s}$ and $I_{s}{ }^{\prime}$ corresponding to $\delta_{w}$ of $2 \pi \times 0.0710 \times 4$ or four times the unit wedge movement. In this experiment we increased the wedge thickness in going from $I_{\text {aft }}$ to $I_{\text {aft }}$ ' 


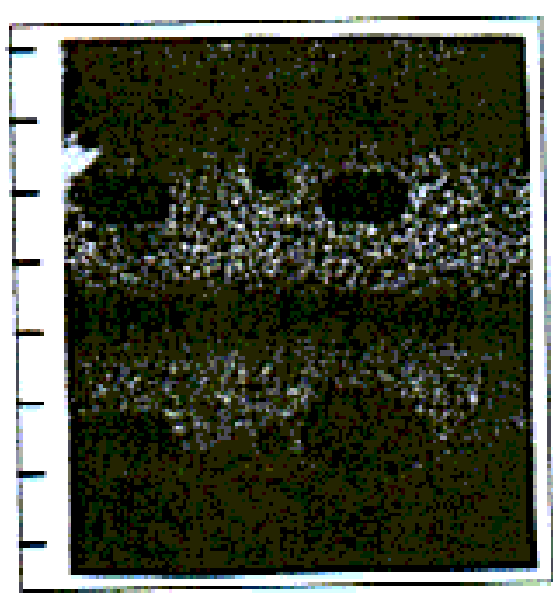

(a) (b)

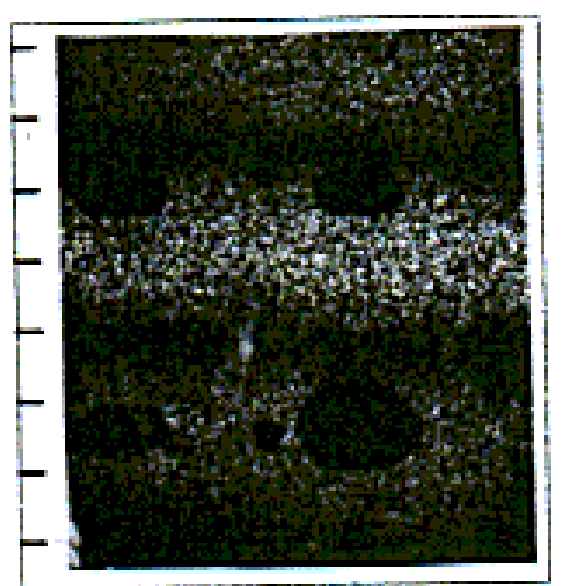

Fig. 3. $I_{s}$ and $I_{s}^{\prime}$ of the model object. The four round dark images are screws.

and rotated the target counterclockwise when viewed from the camera. Thus, in accordance with the explanation above, the fringes in $I_{s}{ }^{\prime}$ are supposed to go down compared with the corresponding fringes in $I_{s}$. Figure 3 clearly shows such a movement. (Note the fringe movement with respect to the position of the screws imaged in these figures.)

Having verified that the fringe moves in the predicted direction, our interest was in determining the accuracy of the movement. Thus we conducted the following experiment: After taking $I_{\text {bef }}$, we moved the wedge in the same direction successively with a constant increment and obtained an image corresponding to $I_{\text {aft }}$ at each wedge position. This enabled us to obtain $I_{s}$ ' at the respective wedge positions. Because these $I_{s}{ }^{\prime}$ terms correspond to the same target movement, the fringes keep moving in the same direction with a constant increment in going from the first wedge position to the last. In the upper part of Fig. 4, we plotted such a movement of two representative fringes in the fringe pattern shown in Fig. 3 in a unit of vertical pixel position and as a function of the unit wedge movement. In the lower part of Fig. 4, the fringe movement is expressed in a unit of fraction of $2 \pi$. The solid lines are theoretical curves representing the unit wedge movement causing a phase change of $2 \pi \times 0.0710$. It is seen that the experimental fringe shift is in fairly good agreement with the theory. The trend line of the experimental data based on least-squares fitting shows a slope of 0.0726 , which agrees with the theoretical value of 0.0710 with an error of only $2.2 \%$.

In summary, we have demonstrated a new algorithm to reveal the order and sign of fringes formed by a dual-beam ESPI setup, where a simple phase shifter such as an ordinary glass wedge can be employed and no calculation is needed. By combining this algorithm with our previously reported phaseextraction method, one can evaluate displacement absolutely and continuously over the entire surface of the object from a total of five frames. This technique

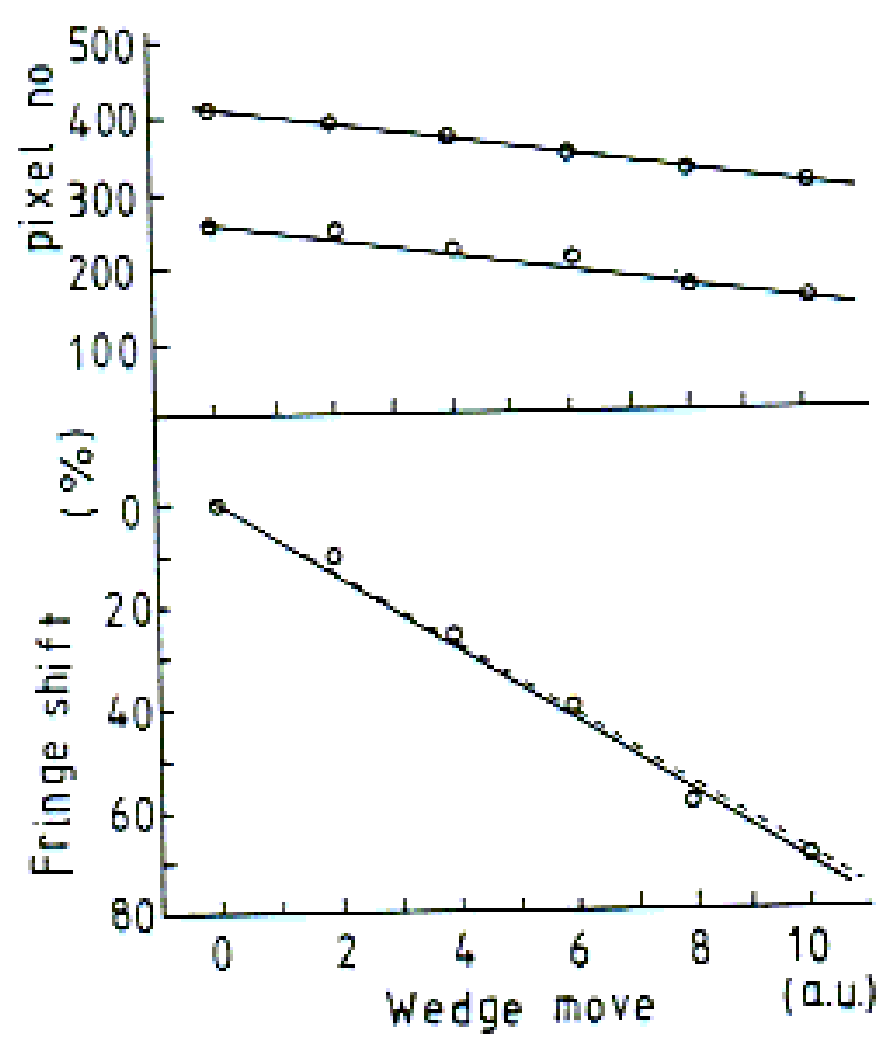

Fig. 4. Fringe shift versus wedge move.

is particularly useful for deformation analyses where displacement is a complicated function of time and space.

\section{References}

1. O. J. Lokberg, "Recent development in video speckle interferometry," in Speckle Metrology, R. S. Sirohi, ed., Vol. 38 of Optical Engincering (Marcel Dekker, New York, 1993), pp. 163-166.

2. S. Yoshida, Y. Hida, V. E. Panin, and L. B. Zuev, in "Application of the wave theory of plastic deformation to a novel scheme of nondestructive analysis of mechanical behavior of solid-state object," in Proceedings of the 10th International Conference on the Strength of Materials, H. Oikawa, K. Maruyama, S. Takeuchi, and M. Yamaguchi, eds. (The Japan Institute of Metals, Sendai, 1994), pp. 295-298.

3. V. E. Panin, "Physical basis of mesomechanics on plastic deformation and fracture of solid-state materials (Russian)," in Physical Mesomechanics and Computer-Aided Design of Materials, V. E. Panin, ed. (Nauka, Novosibirsk, 1995), Vol. 1, pp. 7-28.

4. V. E. Panin, Institute of Strength Physics and Material Science of the Russian Academy of Sciences Siberian Branch, Akademicheski, Tomsk 634055, Russia (personal communication, 1995).

5. D. Kerr, F. Mendoza Santoyo, and J. R. Tyrer, "Extraction of phase data from electronic speckle pattern interferometric fringes using a single-phase-step method: a novel approach," J. Opt. Soc. Am. A 7, 820-826 (1990).

6. G. H. Kaufmann, "Automatic fringe analysis procedures in speckle metrology," in Speckle Metrology, R. S. Sirohi, ed., Vol. 38 of Optical Engineering (Marcel Dekker, New York, 1993), pp. 462-467.

7. S. Toyooka, H. Nishida, and J. Takezaki, "Automatic analysis of holographic and shearographic fringes to measure flexual strains in plates," Opt. Eng. 28, 55-66 (1989).

8. S. Yoshida, Suprapedi, R. Widiastuti, E. T. Astuti, and A. Kusnowo, "Phase evaluation for electronic speckle pattern interferometry deformation analyses," Opt. Lett. 20, 755-757 (1995). 